\title{
TOXICIDAD SUBCRÓNICA Y POSIBLE EFECTO TERATOGÉNICO EN RATAS DEL EXTRACTO ETANÓLICO DE Chuquiraga spinosa (HUAMANPINTA)
}

\author{
Subchronic toxicity and possible teratogenic effect in rats of ethanol extract of Chuquiraga \\ spinosa (huamanpinta) \\ Martín Condorhuamán ${ }^{1}$, Luis A. Rojas ${ }^{1}$, Amadeo Collado ${ }^{1}$, Eliana G. Contreras ${ }^{2}$, Jose A. Ortiz ${ }^{3}$, Javier S. Córdova ${ }^{3}$, Eliberto Ruiz ${ }^{4}$, Oscar \\ Herrera $^{5}$ \\ ${ }^{1}$ Instituto de Investigación en Ciencias Farmacéuticas y Recursos Naturales "Juan de Dios Guevara". ${ }^{2}$ Instituto de Investigación en Química \\ Biológica, Microbiología y Biotecnología, "Marco Antonio Garrido Malo". ${ }^{3}$ Centro Latinoamericano de Enseñanza e Investigación en \\ Bacteriología Alimentaria - CLEIBA". Facultad de Farmacia y Bioquímica. ${ }^{4}$ Facultad de Farmacia y Bioquímica, Universidad Nacional Mayor \\ de San Marcos. ${ }^{5}$ Facultad de Farmacia y Bioquímica, Universidad Nacional San Luis Gonzaga
}

\section{RESUMEN}

El presente estudio tuvo como objetivo determinar la toxicidad subcrónica y posible efecto teratogénico del extracto etanólico de Chuquiraga spinosa (huamanpinta) en ratas de raza Holtzmann. Para la determinación de la toxicidad subcrónica se utilizaron 6o ratas (30 machos y 30 hembras); el primer grupo, control, recibió suero fisiológico $4 \mathrm{~mL} / \mathrm{kg}$ y el segundo grupo $200 \mathrm{mg} / \mathrm{kg}$ de extracto durante cuatro meses consecutivos, realizando la evaluación hematológica y bioquímica al segundo, tercer y cuarto mes. Al final del ensayo (cuarto mes) se realizó el examen anatomopatológico. La determinación del posible efecto teratogénico se realizó utilizando 40 ratas hembras y diez ratas machos. Las ratas hembras recibieron $200 \mathrm{mg} / \mathrm{kg}$ diariamente durante un mes antes del apareamiento y, hasta el nacimiento de las crías, se evaluaron el número implantaciones a nivel de cuernos uterinos, fetos vivos y muertos, y malformaciones morfológicas externas de las crías. Del estudio fitoquímico se determinó que el extracto etanólico presentó taninos, compuestos fenólicos, alcaloides, flavonoides y saponinas. En el ensayo de toxicidad subcrónica se observó que hubo diferencia significativa en los valores hematológicos y bioquímicos al segundo, tercer y cuarto mes de tratamiento y, a nivel histopatológico, no se evidenciaron alteraciones en hígado, riñón y cerebro. En relación al posible efecto teratogénico no se observaron diferencias en el peso de las ratas gestantes, número de implantaciones fetales, número de fetos vivos y alteraciones morfológicas de las crías al momento del nacimiento ni a dos meses de seguimiento. Se concluye que el extracto etanólico no presentó toxicidad subcrónica ni efecto teratogénico a la dosis de $200 \mathrm{mg} / \mathrm{kg}$.

Palabras clave: Toxicidad subcrónica, teratogenico, Chuquiraga spinosa.

\section{SUMMARY}

Objective of this experimental study was to determine the subchronic toxicity and possible teratogenic effect of ethanol extract from Chuquiraga spinosa (huamanpinta) in Holtzman rats. Was used for the determination of subchronic toxicity 60 rats (30 males and 30 females); the first, control, group received saline solution $4 \mathrm{~mL} / \mathrm{kg}$ and the second group extract at $200 \mathrm{mg} / \mathrm{kg}$ during four consecutive months, making evaluation hematological and biochemical the second, third and fourth month. At the end of the trial (fourth month) was made pathological examination. The determination of possible teratogenic effect was performed using 40 female rats and ten male rats. Female rats recived $200 \mathrm{mg} / \mathrm{kg}$ daily by a month before mating and until the birth of brood, was evaluated the number implementations to uterine horns level, live and dead fetuses, and external morphological abnormalities of the offspring. The phytochemical study found that the ethanolic extract showed tannins, phenolic compounds, alkaloids, flavonoids and saponins. In the chronic toxicity test was observed that there was significant difference in hematological and biochemical values to second, third and fourth month of treatment and no histopathological changes in liver, kidney and brain. Regarding to possible teratogenic effect was observed no difference in the weight of pregnant rats, number of fetal implantations, number of live fetuses and morphological abnormalities of the offspring at birth even two months follow up. Was concluded that ethanolic sxtract not showed sub-chronic toxicity or teratogenic effect at $200 \mathrm{mg} / \mathrm{kg}$ dose.

Keywords: Chronic toxicity, teratogenic, Chuquiraga spinosa.

\section{INTRODUCCIÓN}

ए n la actualidad existe abuso de medicamentos herbales de probada o dudosa efectividad, y exagerada confianza en ellos, por lo que su uso implica evidentes peligros, debido a que en su composición fitoquímica existe un conglomerado de compuestos químicos diferentes, farmacológicamente activos, pero con propiedades toxicológicas asociadas. Estos efectos adversos no siempre son conocidos porque no son evidentes a corto o mediano plazo y por la existencia de información no fiable, dispersa y poco asequible, lo que dificulta la búsqueda de evidencias científicas ${ }^{1}$.

Chuquiraga spinosa (huamanpinta) es un arbusto perenne, siempre verde y espinudo de 1 a $1,5 \mathrm{~m}$ de alto, con ramas densas hacia los extremos. Sus hojas son alternas o a veces, las superiores, opuestas, ovaladas a lanceoladas, espinosas en la punta. Sus inflorescencias se presentan en capítulos solitarios, terminales o axilares, sésiles, con el involucro turbinado rojizo o anaranjado, de tres a cuatro cm de largo, formado por brácteas en muchas series. Las cabezuelas están compuestas por 15 a 23 flores de corola anaranjada, con los estigmas sobresaliendo notoriamente 
Tabla 1. Análisis fitoquímico del extracto etanólico de Chuquiraga spinosa.

\begin{tabular}{lcl}
\hline \multicolumn{1}{c}{ Prueba de caracterización } & Resultado & $\begin{array}{l}\text { Metabolito } \\
\text { secundario }\end{array}$ \\
\hline Reacción de la ninhidrina & $(+)$ & $\begin{array}{l}\text { Grupos aminos } \\
\text { libres }\end{array}$ \\
Reacción de la gelatina & $(+)$ & Taninos \\
Reacción con cloruro férrico & $(+)$ & Compuestos fenólicos \\
$\left(\mathrm{FeCl}_{3}\right)$ & $(+)$ & Alcaloides \\
Reacción de Dragendörff & $(+)$ & Alcaloides \\
Reacción de Haeger & $(+)$ & Alcaloides \\
Reacción Mayer & $(+)$ & Flavonoides \\
Reacción de Shinoda & $(+)$ & Quinonas \\
Reacción de Borntrager & $(+)$ & Triterpenos oesteroides \\
Reacción de Lieberman-Burchard & $(+)$ & Saponinas \\
Prueba de la espuma & $(++)$ Abundante cantidad, (++)Regular cantidad, (+) Poca cantidad, (-)Ausente. \\
\hline (++)
\end{tabular}

formando un mechón. Los tallos, hojasy flores seusan como cicatrizantes, sudoríficos, antiinflamatorios, diuréticos, para problemas renales y biliares, y como antisépticos de las vías urinarias y próstata ${ }^{2-4}$. El estudio del extracto etanólico y acuoso evidenció acción antioxidante y antiinflamatoria -in vivo e in vitro-, así como también antifúngica las que se deben a la alta concentración de compuestos fenólicos como flavonoides y derivados del ácido fenólico ${ }^{5}$.

Los estudios fitoquímicos sobre Chuquiraga spinosa indican la presencia de compuestos triterpénicos: lupeol, p-hidroxiacetofenona, 4-(o-(glicosil)hidroxi)acetofenona ${ }^{6}$. El análisis específico de las hojas secadas al aire, evidenciaron compuestos carbonilo (36,1\%): con p-metoxiacetofenona $(8,6 \%)$ y p-hidroxiacetofenona $(5,8 \%)$; e hidrocarburos sesquiterpenos: $\beta$ - humuleno (3,4\%), ar-curcumeno $(2,8 \%)$ y cupareno $(2,5 \%)^{7}$.

La presente investigación tuvo como objetivo evaluar la toxicidad subcrónica y posible efecto teratogénico del extracto etanólico de Chuquiraga spinosa (huamanpinta) administrado por vía oral.

\section{MATERIAL Y MÉTODOS}

La presente investigación experimental de tipo descriptivo, analítico, prospectivo y longitudinal, fue realizada en la Facultad de Medicina de la Universidad Nacional Mayor de San Marcos.

\section{Material biológico}

6o ratas de raza Holtzmann para la determinación de la toxicidad subcrónica, 30 machos con peso de $190 \pm 10$ g y 30 hembras con peso de $160 \pm 10 \mathrm{~g}$.

50 ratas de raza Holtzmann para la determinación del posible efecto teratogénico, 40 hembras con peso de $160 \pm 10 \mathrm{~g}$ y 10 machos con peso de $190 \pm 10 \mathrm{~g}$.

\section{Recolección de la especie vegetal y obtención del extracto etanólico}

Las partes aéreas de Chuquiraga spinosa fueron recolectadas del distrito de Chupaca, provincia de Tambo, región Junín. Se secaron bajo sombra a temperatura no mayor a $40^{\circ} \mathrm{C}$, y se almacenaron en un frasco de vidrio ámbar. El extracto etanólico se obtuvo por el método de maceración, empleando como solvente etanol al 96\%; una vez obtenido el extracto se procedió a evaporar el solvente
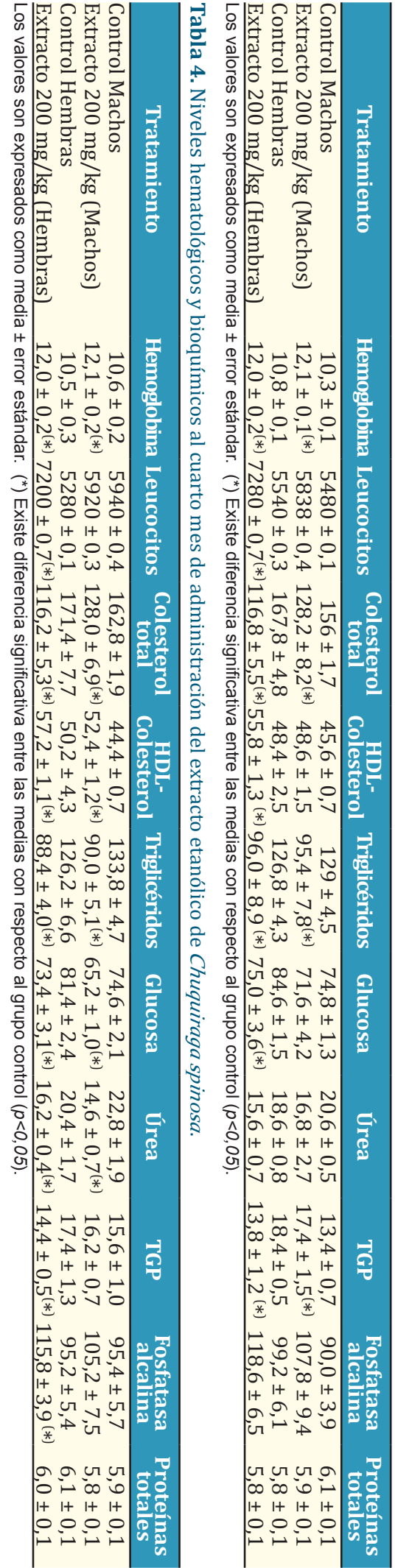
Tabla 5. Niveles de los pesos de las ratas del ensayo de toxicidad subcrónica luego de la administración del extracto etanólico de Chuquiraga spinosa.

\begin{tabular}{lccc}
\hline \multicolumn{1}{c}{ Tratamiento } & Segundo mes & Tercer mes & Cuarto mes \\
\hline Control Machos & $306,4 \pm 1,5$ & $363,2 \pm 3,6$ & $368,2 \pm 2,1$ \\
Extracto 200 mg/kg (Machos) & $296,2 \pm 9,1$ & $327,2 \pm 18,2^{(*)}$ & $297,6 \pm 18,8^{(*)}$ \\
Control Hembras & $243,2 \pm 3,8$ & $267,4 \pm 6,2$ & $260,6 \pm 5,0$ \\
Extracto 200 mg/kg (Hembras) & $221,4 \pm 4,9(*)$ & $246,2 \pm 8,3$ & $246,6 \pm 7,9$ \\
\hline \multicolumn{2}{l}{ Los valores son expresados como media \pm error estándar. $\left({ }^{*}\right)$} & Existe diferencia \\
significativa entre las medias con respecto al grupo control $(p<0,05)$.
\end{tabular}

hasta sequedad completa. En el análisis fitoquímico se procedió a identificar los metabolitos mediante reacciones de coloración y precipitación ${ }^{8}$.

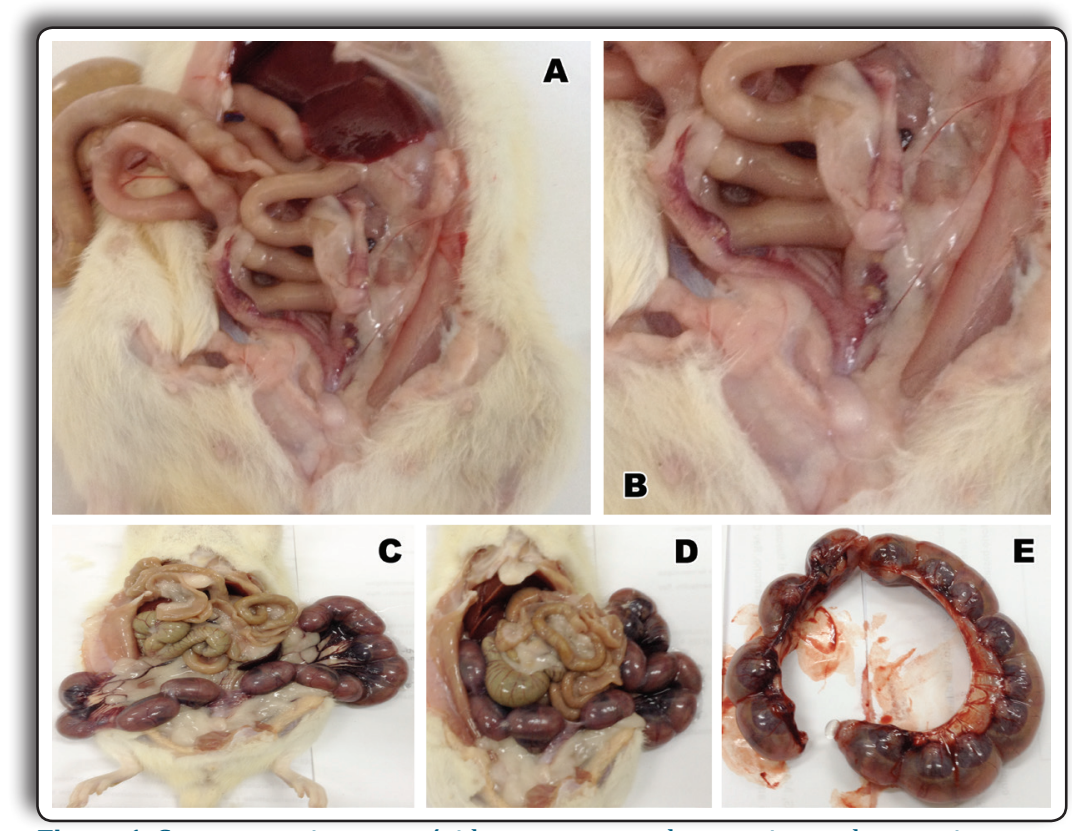

Figura 1. Cuernos uterinos no grávidos grupo control y experimental respectivamente (AyB).Ratas grávidas, en donde se observan cuernos uterinos grávidos del grupo control y experimental (C y D). Cuernos uterinos grávidos aislados del grupo experimental (E).

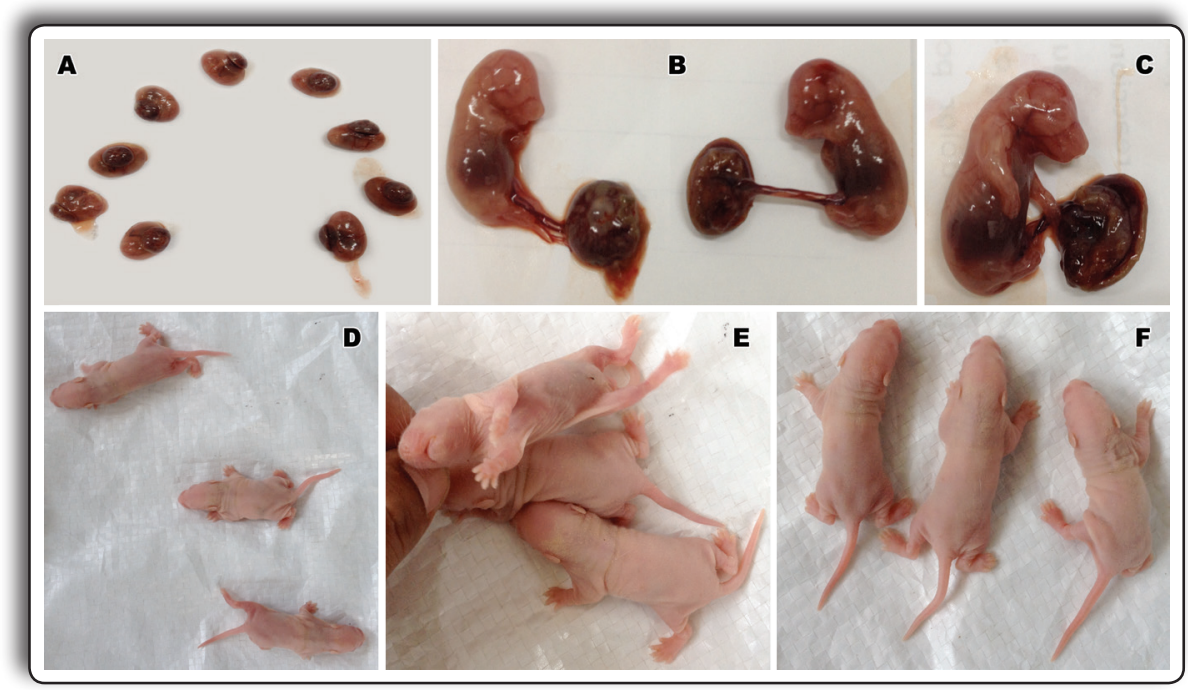

Figura 2. Disección de las bolsas placentarias de ambos grupos (A). Fetos sin alteraciones con sus respectivas placentas (flechas) de ambos grupos (B). Feto de morfología sin alteraciones del grupo experimental (C). Aspectos morfológicos de las crías de ratas del grupo control (D) y grupo experimental (E y F)
Tabla 6. Niveles de los pesos de las ratas gestantes por mes luego de \begin{tabular}{cccc}
\hline Tratamiento & $\begin{array}{c}\text { Primer } \\
\text { mes }^{(*)}\end{array}$ & $\begin{array}{c}\text { Segundo } \\
\text { mes }\end{array}$ & $\begin{array}{c}\text { Tercer } \\
\text { mes }^{(*)}\end{array}$ \\
\hline
\end{tabular}

Control 274,8 $\pm 33,9315,3 \pm 51,6 \quad 314,4 \pm 57,3$

Extracto $200 \mathrm{mg} / \mathrm{kg} 258,8 \pm 42,4 \quad 286,7 \pm 52,0 \quad 272,1 \pm 40,6$ $\left({ }^{*}\right)$ No Existe diferencia significativa entre las medias con respecto al grupo control $(p<0,05)$

\section{Determinación de la toxicidad subcrónica}

Se tuvieron en cuenta las consideraciones dadas por la Norma 408 de la OECD (Guideline for the Testing of Chemicals) y Lagarto et al ${ }^{10}$. Considerando otros estudios realizados, en el presente se trabajó con la dosis $200 \mathrm{mg} / \mathrm{kg}$.

Se utilizaron 60 ratas albinas que se agruparon aleatoriamente en dos grupos (cada uno con 30 ratas entre machos y hembras). El primer grupo (control) recibió suero fisiológico 4 $\mathrm{mL} / \mathrm{kg}$ y el segundo extracto etanólico $200 \mathrm{mg} /$ $\mathrm{kg}$. El extracto se administró una vez al día por vía oral durante 120 días. Se observo la evolución del peso corporal y los posibles signos de toxicidad (caída de pelo, cambio de coloración en los ojos, agresividad o pasividad); cada 30 días se extrajo sangre por punción cardiaca de cinco machos y cinco hembras decadagrupoen busca decambios a nivel hematológico y bioquímico en el segundo, tercer y cuarto mes. Sólo al final del ensayo (cuarto mes) se hizo el estudio histopatológico de hígado, riñón y cerebro.

\section{Determinación del posible efecto teratogénico}

El estudio se realizó utilizando 40 ratas hembras y diez ratas machos. Antes de colocar a las hembras en las jaulas con los respectivos machos, se les administró a todas $200 \mathrm{mg} / \mathrm{kg}$ del extracto etanólico diariamente por 30 días, luego de lo cual se propició el apareamiento a razón de cuatro hembras por macho, donde se comprobó la cópula por lavado vaginal con suero fisiológico, observándose al microscopio óptico la presencia de espermatozoides en la lámina, considerándose como día uno de la gestación. Las ratas gestantes se mantuvieron en jaulas independientes con libre acceso al agua y comida, con un ritmo de 12 horas de luzy oscuridad, y se pesaron en días alternos. Estas ratas recibieron $200 \mathrm{mg} / \mathrm{kg}$ hasta el día 19 de gestación. Se evaluó el número de implantaciones, número de fetos vivos y muertos, y alteraciones morfológicas externas de las crías nacidas ${ }^{11}$.

\section{Consideraciones éticas}

Se respetaron las normas establecidas por la guías de mantenimiento y conservación de animales de experimentación ${ }^{\mathbf{1 2}}$. 


\section{Análisis estadístico}

Los resultados fueron analizados mediante estadística descriptiva y el test de Student (media y error estándar) y se aplicó el test de Tukey para las comparaciones entre los grupos, considerándose estadísticamente significativo para un $p<0,05$.

\section{RESULTADOS}

Se presentan de la siguiente manera: análisis químico, tabla 1; estudio de la toxicidad subcrónica, tablas 5-6; evaluación de posibles efectos teratogénicos, figuras 1 y 2.

\section{DISCUSIÓN}

Uno de los problemas más frecuentes asociados con el uso de plantas medicinales es la ausencia de evidencias farmacológicas, toxicológicas y clínicas ${ }^{13}$. La existencia de plantas con elevado potencial terapéutico constituye una alternativa farmacológica de marcado interés en el tratamiento de muchas enfermedades, de ahí la importancia de realizar estudios preclínicos con el propósito de detectar posibles efectos tóxicos post administración.

Dentro de la batería de ensayos de primera barrera se encuentran los estudios de toxicidad a dosis única, imprescindibles en la estimación del potencial tóxico de una sustancia, referidos como estudios cuali-cuantitativo de los fenómenos tóxicos y de su aparición en función del tiempo tras la administración de una dosis única de la sustancia o de varias dosis fraccionadas en el transcurso de 24 horas ${ }^{14}$.

La Organización Mundial de la Salud apoya el uso adecuado de medicamentos a base de plantas y promueve el uso de los recursos que han demostrado ser seguros y eficaces ${ }^{15}$. Algunas plantas medicinales han resistido la prueba científica, pero otras son utilizadas sólo por razones tradicionales para proteger, restaurar o mejorar la salud, necesitando ser estudiadas a pesar de la experiencia registrada con su uso tradicional en los últimos años ${ }^{16}$.

El extracto etanólico de Chuquiraga spinosa presentó taninos, compuestos fenólicos, alcaloides, flavonoides, quinonas, triterpenos o esteroides, y saponinas, también descritas por Galvez ${ }^{6}$, que en conjunto es posible que hayan presentado antagonismo en los efectos de la toxicidad crónica.

Sobre el análisis estadístico, se observó que hubo diferencia significativa en los valores hematológicos $\mathrm{y}$ bioquímicos al segundo, tercer y cuarto mes de administracióndel extractoetanólico, peroaparentemente no denotaron toxicidad del extracto porque se encuentran dentro rangos normales (tablas 2, 3 y 4$)^{17}$. Hubo reducción de los niveles de colesterol total y triglicéridos (efecto hipocolesterolemiante e hipotriglicerimiante), aumento de HDL colesterol (posible efecto cardioprotector), aumento de leucocitos dentro de los rangos normales (posible efecto inmunomodulador), reducción de la glucosa (efecto hipoglicemiante) y a nivel hepático disminución de TGP y aumento de la fosfatasa alcalina (hepatoprotección con posible efecto colestásico).
No se evidenciaron alteraciones en el examen anatomopatológico al cuarto mes de tratamiento en los órganos evaluados, lo que contrasta con lo encontrado por la hematología y bioquímica. En la parte clínica los animales no presentaron caída de pelo, cambio de coloración en los ojos, ni agresividad o pasividad marcadas durante el estudio.

En los ensayos de toxicidad subcrónica, se observó que los animales de experimentación que recibieron extracto etanólico sufrieron disminución de sus pesos en relación al grupo control con significancia estadística, sobre todo los machos en el tercer y cuarto mes, sin embargo, siempre estuvieron dentro de los parámetros normales de acuerdo al sexo del animal ${ }^{18,19}$.

En relación al posible efecto teratogénico con la dosis $200 \mathrm{mg} / \mathrm{kg}$ de extracto, no se observaron cambios ni alteraciones en el peso de las ratas gestantes (tabla 6), número de implantaciones, número de fetos vivos y no hubieron alteraciones morfológicas de la crías al momento del nacimiento (figura 1 y 2), ni a dos meses de seguimiento en su desarrollo.

\section{CONCLUSIONES}

El extracto etanólico de Chuquiraga spinosa presenta como metabolitos taninos, compuestos fenólicos, alcaloides, flavonoides, quinonas, triterpenos o esteroides, y saponinas.

No presentó toxicidad subcrónica a niveles hematológico, bioquímico ni histopatológico en ratas no grávidas, a la dosis de $200 \mathrm{mg} / \mathrm{kg}$.

El extracto etanólico administrado a las ratas grávidas a dosis de $200 \mathrm{mg} / \mathrm{kg}$, no presentó cambios en el peso ni en el número de implantaciones y no evidenció efecto teratogénico en los fetos, ni alteraciones morfológicas de la crías al momento del nacimiento ni a dos meses de seguimiento.

\section{Agradecimiento}

Proyecto financiado por el VRI-UNMSM, Código de Proyecto № 140401041

\section{REFERENCIAS BIBLIOGRÁFICAS}

1. Domínguez A, Reyes L, García O, Macías B, Pérez L. Informática médica y toxicología de plantas medicinales con acción antimicrobiana. Ciencia en su PC. 2006; 2: 1-19.

2. Mostacero J, Mejía F. Taxonomía de Fanerógamas Peruanas. ${ }^{\text {ra }}$ ed. Trujillo: Editorial Libertad, 1993.

3. Orrego F, Watson J, Flores A, Rojas G. Flores Silvestres de Chile. Flores del Norte Grande. Santiago de Chile: Quad/ Graphics S.A; 2013.

4. Bussmann R, Glenn A, Sharon, D. Antibacterial activity of medicinal plants of Northern Peru - can traditional applications provide leads for modern science?. Indian J Tradit Knowle. 2010; 9(4): 742-53.

5. Casado R, Landa A, Calvo J, García-Mina JM, Marston A, Hostettmann K, et al. Anti-inflammatory, antioxidant and antifungal activity of Chuquiraga spinosa. Pharm Biol. 2011; 49(6): 620-6. 
6. Galvez M. Estudio Fitoquímico de la Chuquiraga spinosa. Revista de Química. PUCP. 1996; X(1): 131-7.

7. Senatore F. Composition of the essential oil of Chuquiraga spinosa (R. et P.) D. Don. Flavour and Fragrance Journal. 1996; 11(4): 215-7.

8. Lock de Ugaz O. Investigación fitoquímica - Métodos en el estudio de productos naturales. Lima: Fondo Editorial de la Pontificia Universidad Católica del Perú; 1988.

9. OECD. Test No. 408: Repeated Dose 90-Day Oral Toxicity Study in Rodents, OECD Publishing, Paris. 1998. DOI: http://dx.doi.org/10.1787/9789264070707-en

10. Lagarto A, Tillan J, Bueno V, Chávez I, Guerra I, Vega Y, et al. Toxicidad aguda oral y subcrónica en ratas de un extracto acuoso liofilizado de Ocimum tenuiflorum L. Revista de Toxicología. 2005; 22(3): 175-9.

11. Díaz S, Ruíz C. Estudio teratogénico del gabapentin en ratones. Revista Científica Juvenil, 20o6; V: 75-80.

12. Olfert ED, Cross BM, McWilliam AA. Guide to the care and use of experimental animals. Otawa: Canadian Council on Animal Care; 1993.

13. Olaya M, Lozano M, Botero L, Rincón V, Guerrero P. Evaluación de la toxicidad oral aguda y sub-crónica del extracto etanólico de la especie Valeriana pavonii en ratas Wistar. Colomb Med. 2010; 41(3): 256-66.

14. Bermudez D, Monteagudo E, Boffill M, Díaz L, Roca A, Betancourt E, et al. Evaluación de la toxicidad aguda de extractos de plantas medicinales por un método alternativo. Revista Electrónica de Veterinaria [Internet]. 2007 [Citado 12 abril 2015]; VIII(3): 1-7. Disponible en: http://www.redalyc.org/articulo.oa?id=63613302006

15. Organización Mundial de la salud. Estrategia de la OMS sobre medicina tradicional 2002-2005. Ginebra: Renata Kerr Design; 2002.

16. Rojas J, Díaz D. Evaluación de la toxicidad del extracto metanólico de hojas de Passiflora edulis Sims (maracuyá), en ratas. An Fac med, 2009; 70(3): 175-80.

17. Arroyo J, Rojas J, Chenguayen J. Manual de Modelos Experimentales de Farmacología. Lima: Publicaciones ASDIMOR; 2004.

18. Pahl PJ. Growth curves for body weight of the laboratory rat. Aust J Biol Sc. 1969; 22: 1077-80.

19. Cossio M, Gómez R, Vargas R, Hochmuller R, Arruda M. Curvas de referencia para valorar el crecimiento físico de ratas machos Wistar. Nutr Hosp, 2013; 28(6): 2151-6.

Manuscrito recibido el: 17/03/2016

Aceptado para su publicación el: 19/09/2016

\section{Correspondencia}

Nombre: Martín Condorhuamán Figueroa

Dirección: Jr. Puno 1002 - Lima

e-mail: marcofi71@yahoo.es 